\title{
TRAIL Suppresses Human Breast Cancer Cell Migration via MADD/CXCR7
}

\author{
Rui Wang, Jin-Cheng Li*
}

\begin{abstract}
Background: Tumor necrosis factor-related apoptosis-inducing ligand (TRAIL) can specifically induce apoptosis limited to various cancer cells, so this reagent is considered a promising medicine for cancer therapy. TRAIL also exerts effects on non-apoptotic signals, relevant to processes such as metastasis, autophagy and proliferation in cancer cells. However, the mechanisms of TRAIL-regulated non-apoptotic signals are unclear. The purpose of this study was to investigate MADD/CXCR7 effects in TRAIL-mediated breast cancer cell migration. Materials and Methods: The ability of MADD/CXCR7 to regulate MVPsignaling in TRAIL-mediated breast cancer cells migration was evaluated by transwell migration assay, quantitative RT-PCR, Western blotting and knock down experiments. Results: In this study, we found that treatment with TRAIL resulted in induced expression levels of MADD and CXCR7 in breast cancer cells. Knock down of MADD followed by treatment with TRAIL resulted in increased cell migration compared to either treatment alone. Similarly, through overexpression and knockdown experiments, we demonstrated that CXCR7 also positively regulated TRAILinhibited migration. Surprisingly, knock down of MADD lead to inhibition of TRAIL-induced CXCR7 mRNA and protein expression and overexpression of CXCR7 lead to the reduction of MADD expression, indicating that MADD is an upstream regulatory factor of TRAIL-triggered CXCR7 production and a negative feedback mechanism between MADD and CXCR7. Furthermore, we showed that CXCR7 is involved in MADD-inhibited migration in breast cancer cells. Conclusions: Our work defined a novel signaling pathway implicated in the control of breast cancer migration.
\end{abstract}

Keywords: TRAIL - MADD - CXCR7 - migration

Asian Pac J Cancer Prev, 16 (7), 2751-2756

\section{Introduction}

TNF-related apoptosis-inducing ligand (TRAIL) is a member of the tumor necrosis factor (TNF) superfamily, and bears the advantage of being able to kill tumor cells without harming normal cells (Shi et al., 2003; Kruyt, 2008; Attar et al., 2014). TRAIL evokes its response by binding to and inducing trimerization of membrane death receptor DR4 and DR5 as an trimer which is its active form, the death domain of DR4 and DR5 aggregates and is thus activated, FADD and procasepase- 8 are then recruited to form the DISC which leads to activation of caspase- 8 and initiation of apoptosis (Falschlehner et al., 2007; Szliszka et al., 2009; Nogueira et al., 2014). In addition, TRAIL can also activate non-apoptotic (non-canonical) signals that can enhance the inflammatory, survival, proliferation and cell migratory responses in different cell types (Azijli et al., 2013). Various kinases have been reported play an important role in TRAIL-induced noncanonical signals (Azijli et al., 2012).

Insulinoma-Glucagonoma-20 (IG-20) gene is located on chromosome 11p11 and contains 36 exons, currently 7 splicing isoforms have been identified and 4 forms are expressed in human, which are IG20, MADD, IG20-SV2 and DENN-SV (Efimova et al., 2004). MADD was first cloned by Schievella AR et al in 1997 and was recognized for MAP kinase-Activating Death Domain protein (Schievella et al., 1997). This protein is expressed at much higher levels in cancer cells and tissues relative to their normal counterparts (Al-Zoubi et al., 2001). Up to now, DENN-SV draw a lot of attention in it's antiapoptotic role. MADD can directly interact with TNFR1 cytoplasmic tail and promotes TNF $\alpha$-induced activation of MAPK and JNK (Al-Zoubi et al., 2001). MADD also facilitate the recruitment of Grb2 and Sos $1 / 2$ to the TNFR1 complex with resultant Ras activation (Kurada et al., 2009). Interestingly, a recent study showed that MADD is overexpression in breast cancer tissues and MADD knockdown enhances TRAIL induced apoptosis in breast cancer cells, implying that MADD is related to breast cancer progression (Turner et al., 2013).

CXCL12, also called stromal cell-derived factor-1, is a member of the CXC subfamily of chemokines and express in avariety of tissues (Aiuti et al., 1997; Pablos et al., 1999). CXCR4 and CXCR7, which belong to the family of G-protein coupled receptors (GPCRs), is two known 
receptors for CXCL12 (Tachibana et al., 1998; Burns et al., 2006). CXCL12/CXCR4 signaling has been reported to promote growth of primary tumors and progression to metastatic disease in breast and lung cancer (Kakinuma and Hwang, 2006). Similar with CXCR4, CXCR7 is also expressed in different human cancers, and mediates a broad range of cellular activities, including proliferation, survival, and adhesion by binding with CXCL12 (Burns et al., 2006). However, the function and mechanism of CXCR7 in metastasis is still unclear and controversial. A study suggested CXCR7 play no role in bare filter migration (Thelen and Thelen, 2008). Another study suggested that down regulation of CXCR7 expression by using a CXCR7 shRNA significantly inhibits SMMC-7721 cells invasion, adhesion and angiogenesis (Zheng et al., 2010). Moreover, a recent study has shown that CXCR4 mediating breast cancer invasion and CXCR7 impairing invasion but enhancing primary tumor growth through angiogenesis (Hernandez et al., 2011).

Although, there is increasing evidence that MADD and CXCR7 may participate in tumor development, not much is currently known about the role of MADD and CXCR7 in the migration of human breast cancer. In this study, we set out to dissect the roles of MADD and CXCR7 in TRAIL inhibited breast cancer cells migration. Our results showed that TRAIL treatment inhibits the migration of breast cancer cells by a heretofore unrecognized mechanism, in which TRAIL treatment stimulates MADD and CXCR7 expression, and TRAIL treatment stimulates CXCR7 expression through MADD.

\section{Materials and Methods}

\section{Cell culture}

MDA-MB-231 cells were cultured in Dulbecco's modified Eagle's medium (Gibco BRL, Grand Island, NY) supplemented with $10 \%$ fetal bovine serum (Gibco BRL), 2 mM L-glutamine (Invitrogen). SKBR3 cells were cultured in RPMI 1640 medium supplemented $10 \%$ fetal bovine serum (Gibco BRL). T47D cells were cultured in DMEM medium supplemented $10 \%$ fetal bovine serum (Gibco BRL). All cell lines were cultured with $100 \mathrm{U} /$ $\mathrm{ml}$ penicillin, and $100 \mu \mathrm{g} / \mathrm{ml}$ streptomycin sulfateand maintained at $37^{\circ} \mathrm{C}$ in a $5 \% \mathrm{CO}_{2}$ incubator. All these cell lines were original purchased from cell bank of the Chinese Academy of Science, Shanghai.

\section{Antibodies and reagents}

Antibodies against CXCR7 and $\beta$-actin were purchased from Sigma (St. Louis, MO, USA). The donkey anti-rabbit and anti-goat peroxidase-conjugated polyclonal secondary antibody were purchased from GE Health care (GE int. USA). Human TRAIL was purchased from Peprotech (Peprotech, Rocky Hill, NJ, USA). TRIzol, Lipfectamine-2000, Enzyme MIX were purchased from Invitrogen (Basel, Switzerland). pRNAi-U6.1/Neo and TOP10 competent E.coli were purchased from Biomics Biotechnology (Co., Ltd., Jiangsu).

Lentivirus production and transduction Lentiviruses were generated and used to transduce
MDA-MB-231 and T47D cells as described previously (Turner et al., 2013). Virus encoding scrambled shRNA (C) served as a control, virus encoding 13L-shRNA (13L) targets exon 13L expressed only in IG20pa and MADD isoforms of the IG20 gene, and virus encoding 16E-shRNA (16E) expressed only in IG20pa and IG20$\mathrm{SV} 2$ isoforms of the IG20 gene. The least amount of viral supernatant required to transduce $70 \%$ of target cells without apparent cytotoxicity was the optimal viral titer and was used in subsequent experiments. We routinely obtained transduction efficiencies of at least $90 \%$ as monitored by green fluorescent protein fluorescence using fluorescence-activated cell sorting.

\section{RNAi experiments and transfection}

RNAi experiments were performed as described (Wang et al., 2014). Briefly, Double-strand oligonucleotides corresponding to the target sequences were cloned into pGPU6/Neo vector containing the human U6 promoter (GenePharma, Shanghai, China). All DNA oligonucleotides were synthesized by Invitrogen (Basel, Switzerland). The target sequences for human CXCR7 mRNA sequence (accession no. NM_020311) are as follows: \#1, 5'-GCATCTCTTCGACTACTCAGA -3'; \#2, 5'-CACTATTGGTGTACCTTATAA-3'; \#3 5' - CCGGAA GATCATCT TC TCCTA-3'. The target sequences for control are as follows: 5'-GTTCTCCGAACGTGTCACGT-3'. Cells were transfected by using Lipofectamine 2000 (Invitrogen) according to the manufacturer's protocol.

\section{RNA extraction and Quantitative RT-PCR analysis}

Total RNA was isolated using TRIzol (Invitrogen, Basel, Switzerland) according to the manufacturer's protocol. Measure the absorbance at $260 \mathrm{~nm}$ to determine the concentration of total RNA. $1 \mathrm{mg}$ of RNA of each sample was reversely transcribed to cDNA by One Step Primerscript miRNA cDNA Synthesis kit (Takara, Dalian, China). Quantitative RT-PCR (real-time PCR) was performed using a LightCycler 480 (Roche) and the SYBR Green system (Applied Biosystems). GAPDH was amplified as an internal control. Real-time RT-PCR primers were as follows: GAPDH sense, 5'GGAAGGTGAAGGTCGGAGTCAACGG-3'; antisense, 5'- CTCGCTCCTGGAAGATGGTGATGGG-3'. CXCR7 sense, 5'-TGGTGGACACGGTGATGTG-3', antisense, 5'-AAATGCTGCCGAAGAGGTT-3'.

\section{Reverse transcription and PCR}

A $1 \mu \mathrm{g}$ sample of total RNA was used for reverse transcription using Super-Script One-Step RT-PCR system (Invitrogen) and random primer. cDNAs were synthesized at $37^{\circ} \mathrm{C}$ for 45 minutes followed by incubation at $94^{\circ} \mathrm{C}$ for 5 minutes. Subsequently, 28 cycles of PCR were carried out with denaturation at $95^{\circ} \mathrm{C}$ for 30 seconds, annealing at $55^{\circ} \mathrm{C}$ for 30 seconds and extension at $72^{\circ} \mathrm{C}$ for 30 seconds; followed by a final incubation at $72^{\circ} \mathrm{C}$ for 10 minutes. The sequences of F2-B2 primers have been previously reported (Al-Zoubi et al., 2001). The $\beta$-actin primers is: F: 5'-GGACTTCGAGCAAGAGATGG-3'; R:5'-AGGAAGGAAGGCTGGAAGAG-3'. The PCR 
products were separated on a $2 \%$ agarose gel which allows for the identification of each of the four IG20 isoforms.

\section{Transwell migration assay}

The ability of cells to migrate was assayed by using transwell inserts (24-well insert, pore size $8 \mathrm{~mm}$; Corning, Inc., Corning, NY). After Cells were treated with TRAIL or transfected with indicated plasmid, the cells were harvested and added to the upper chamber. Simultaneously, the lower chambers were filled with $10 \%$ FBS as a chemoattractant. After $18 \mathrm{~h}$ of incubation at $37^{\circ} \mathrm{C}$ with $5 \% \mathrm{CO} 2$, the number of cells that migrated through the pores was counted in eight microscopic fields by a microscope (IX71, Olympus, Japan) at 40× magnification. The software used to count migrated and non-migrated cells was Image-Pro Insight (Version 8.0.21). The mean number of migrating cells was expressed as a percentage relative to the control, which was designated as $100 \%$.

\section{Western blot}

For the preparation of lysates, the cells were washed with ice-cold PBS solution and lysed in lysis buffer (50 mM Tris-HCl, pH7.4, 150 mM NaCl, 1 \% Nonidet P-40, and $0.1 \%$ SDS supplemented with protease inhibitors). The protein concentration was determined with the BioRad protein assay reagent according to the Bradford method. Samples were loaded to the prepared $12 \%$ PAGE analysis, and electrophoretically transferred to PVDF membrane (Millipore, USA). After blocking, Membranes were then incubated with primary antibody at $4{ }^{\circ} \mathrm{C}$ on a shaker overnight, and washed 3 times, $5 \mathrm{~min}$ each. Peroxidase conjugated second antibody was then added at 1:1000, incubated for $2 \mathrm{~h}$, and washed 3 times, $5 \mathrm{~min}$ each. The band was visualized by ECL (Amersham Biosciences, USA).

\section{Statistical analyses}

All experiments were performed in duplicate or quadruplicate. Statistical analysis was performed using the two-tailed Student $t$ test for comparison between two groups. A P value of $<0.05$ was considered statistically significant.

\section{Results}

\section{TRAIL-inhibited breast cancer cells migration through $M A D D$}

It has been reported that TRAIL inhibits MDAMB-231 cell migration (Wang et al., 2013), so we first examine the effect of TRAIL on the migration of other two breast cancer cell lines. As shown in Figure $1 \mathrm{~A}-\mathrm{C}$, TRAIL treatment suppressed the migration of T47D, SKBR3 and MDA-MB-231 cells. Recently, an interesting study has shown that, in four IG20 isoforms, only DENN-SV and MADD expressed in MCF-7 and T47D cells and 13L-shRNA targeted exon 13L and selectively down-modulated MADD in two breast cancer cell lines (Turner et al., 2013). To test their efficiency of shRNAs described in the reference, we repeat the experiment. As shown in Figure $1 \mathrm{D}$ and E, 13L-shRNA selectively down-modulated MADD, but not DENN-SV. And, the
16E-shRNA, that specifically targets exon 16, had no apparent effect on expression of MADD and DENN-SV (Figure $1 \mathrm{D}$ and $\mathrm{E}$ ). To determine the effects of TRAIL on the MADD expression, T47D cells were treat with or without TRAIL (10 ng/ml) for 24 hour. Results from RTPCR using F2-B2 primers showed that relative mRNA levels of MADD were much higher in TRAIL treatments MCF-7 cells (Figure $1 \mathrm{~F}$ ).

It has been reported that MADD knockdown in breast cancer cells results in spontaneous apoptosis (Turner et al., 2013), however, no further studies have been investigated the role of MADD in the migration of breast cancer cells. To highlight this question, we investigate the role of MADD knockdown in the migration of T47D cells. As determined by transwell migration assay, targeting exon 13L with 13L-shRNA resulted in the migration of T47D cells (Figure $1 \mathrm{G}$ ). We also investigated the role of MADD in the regulation of T47D cell migration mediated by TRAIL. Results from transwell migration assay showed that the TRAIL-reduced T47D cell migration was consistently induced in the presence of 13L-shRNA, but
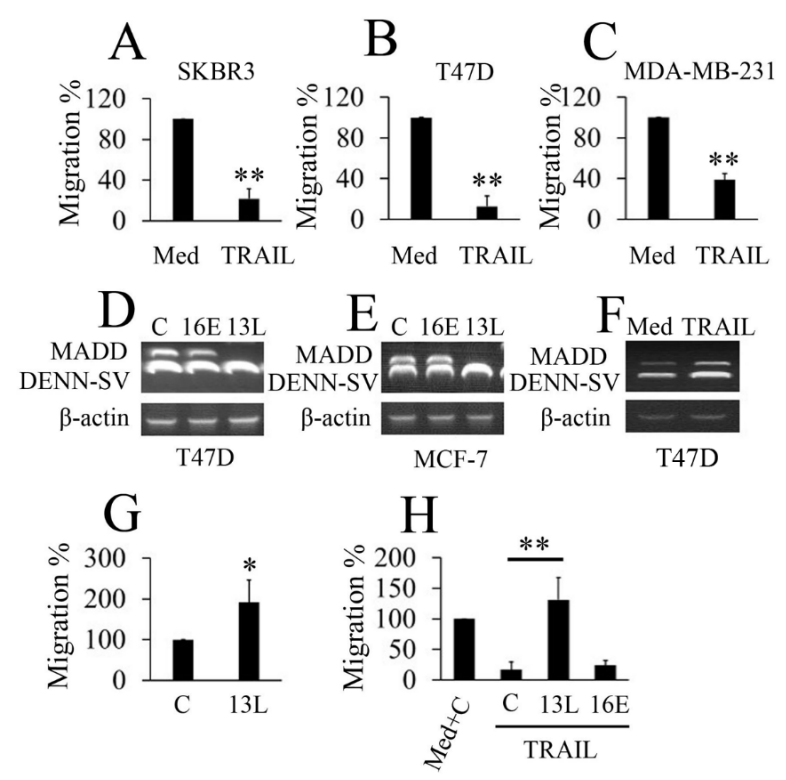

Figure 1. Determination of the Effect of MADD on the Regulation of Breast Cancer Cells Migration Mediated by TRAIL. (A) SKBR3 cells were pre-treated with TRAIL for 1 hour at a concentration of $10 \mathrm{ng} / \mathrm{ml}$, followed by culturing in a transwell system for 18 hour. (B, C) Experiments were performed as in A except T47D (B) or MDA-MB-231 (C) cells were used. (D, E) T47D (D) and MCF-7 (E) cells were transduced with the indicated virus for 72 hour at which point RNA was extracted and used (1 mg) for RT-PCR using F2-B2 primers. (F) T47D cells were treat with TRAIL for 24 hour at a concentration of 10 $\mathrm{ng} / \mathrm{ml}$. RNA was extracted and used $(1 \mathrm{mg})$ for RT-PCR using F2-B2 primers. (G) T47D cells were infected with the indicated shRNA expressing lentiviruses for 56 hours and allowed to migrate towards serum for 18 hours. (H) T47D cells were infected with the indicated shRNA expressing lentiviruses for 55 hours. Then pre-treated with TRAIL $(10 \mathrm{ng} / \mathrm{ml})$ for 1 hour and allowed to migrate towards serum for 18 hours. In the transwell migration assay, the data were expressed as a percentage of the control. In the real-time RT-PCR experiments, the control was designated as 1 . All experiments were repeated at least three times with similar results. Bar graphs represent means $\pm \mathrm{SD}, \mathrm{n}=3$ $(* * p<0.01 ; * p<0.05)$ 
Rui Wang and Jincheng $\mathrm{Li}$

not affected by 16E-shRNA (Figure $1 \mathrm{H}$ ). Together, these results demonstrate that reduction of breast cancer cells migration regulated by TRAIL may require MADD.

TRAIL-inhibited breast cancer cells migration through CXCR7.

It has reported that TRAIL inhibits CXCR4 expression and ultimately limits the migration of breast cancer cells (Wang et al.,2013). Another study has shown that CXCR4 mediating breast cancer invasion and CXCR7 impairing invasion (Hernandez et al., 2011). In light of these two reports, we investigated whether CXCR7 was involved in the regulation breast cancer cells migration. We designed three RNAi plasmids for CXCR7 (CXCR7-RNAi \#1, \#2 and \#3) (Figure 2A). In cell migration experiments, CXCR7 knockdown by CXCR7-RNAi \#1 promoted migration in T47D cells, whereas CXCR7-RNAi \#2 had little effect due to low efficiency (Figure 2B). Conversely, overexpression of CXCR7 strongly inhibited migration in T47D cells (Figure 2C). We also investigate the effect of TRAIL on CXCR7 expression. Results from real-time RT-PCR and western blot analyses confirmed that TRAIL significantly induced CXCR7 expression in T47D cells

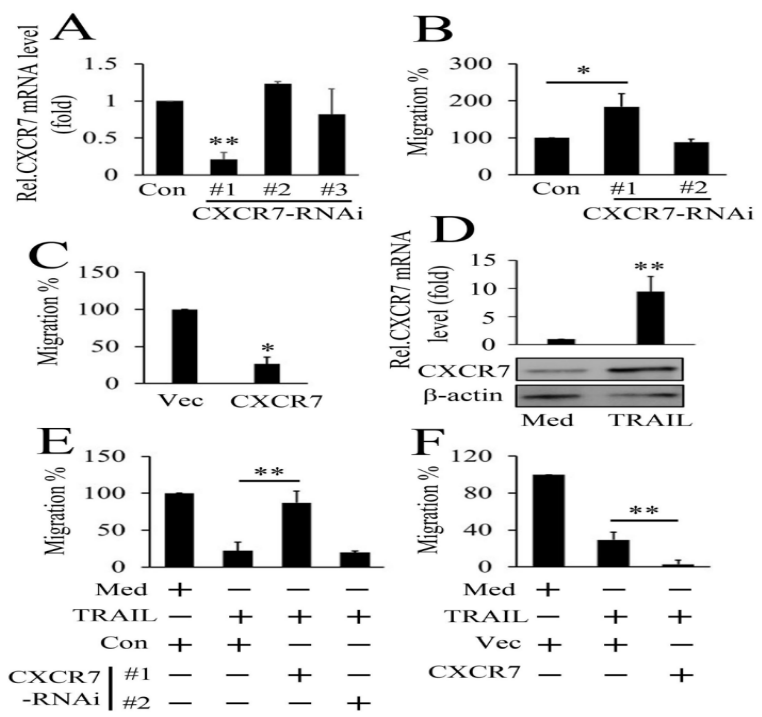

Figure 2. Determination of the Effect of CXCR7 on the Regulation of T47D Cells Migration Mediated by TRAIL. (A) T47D cells were transfected with shRNAcontrol or indicated CXCR7-RNAi for 48 hours. CXCR7 mRNA level was detected by real-time RT-PCR analyses. (B) T47D cells were transfected with shRNA-control or indicated CXCR7-RNAi for 30 hours and allowed to migrate towards serum for 18 hours. (C) Experiments were performed as in B except T47D cells were transfected with pcDNA3.1-Mock (empty vector) or pcDNA3.1-CXCR7. (D) T47D cells were treat with TRAIL for 24 hour at a concentration of $10 \mathrm{ng} / \mathrm{ml}$. CXCR7 mRNA (upper panel) and protein (lower panel) were detected by real-time RT-PCR and western blot, respectively. (E) T47D cells were transfected with shRNA-control or indicated CXCR7-RNAi for 30 hours. Then pre-treated with TRAIL (10 $\mathrm{ng} / \mathrm{ml}$ ) for 1 hour and allowed to migrate towards serum for 18 hours. (F) Experiments were performed as in B except T47D cells were transfected with pcDNA3.1-Mock (empty vector) or pcDNA3.1-CXCR7. All experiments were repeated at least three times with similar results. Bar graphs represent means $\pm \mathrm{SD}, \mathrm{n}=3$ $\left(* * \mathrm{P}^{*}<0.01 ; * \mathrm{P}<0.05\right)$
(Figure 2D). Because both TRAIL and CXCR7 can regulate cell migration, we examined whether CXCR7 is involved in TRAIL-inhibited migration. Using transwell migration assay, we showed that CXCR7 expression knockdown inhibited TRAIL-mediated reduction of migration and CXCR7 overexpression stimulated TRAILmediated reduction of migration (Figure $2 \mathrm{E}$ and $\mathrm{F}$ ). Taken together, these data suggest that TRAIL-reduced breast cancer cells migration is mediated through CXCR7.

\section{TRAIL induces CXCR7 in MADD-dependent manner and} CXCR7 in turn inhibit MADD expression

To confirm the influence of MADD on the TRADD induced CXCR7 expression, we performed real-time RT-PCR assays, which demonstrated that TRAILinduced CXCR7 activation was reduced by transducing with 13L-shRNA (Figure 3A, upper panel). CXCR7 protein levels showed a similar trend as determined by western blot (Figure 3A, lower panel). To identify the role of CXCR7 in the expression of MADD, T47D cells were transfected with CXCR7 overexpression plasmid or control vector. Results from RT-PCR using F2-B2 primers confirmed that MADD mRNA levels were downregulated by CXCR7 (Figure 3B). Conversely, knockdown of CXCR7 induced MADD mRNA levels in T47D cells (Figure 3C). In conclusion, these data suggest that TRAIL activates CXCR7 expression through MADD; and conversely, CXCR7 suppress MADD expression.

\section{$M A D D$-inhibited breast cancer cells migration through CXCR7}

To define the role of CXCR7 in the regulation of MADD reduced migration, T47D cells were transduced with the indicated virus (13L or control) and then transfected with indicated RNAi plasmids for CXCR7. As shown in Figure 4A, transduction with 13L-shRNA and
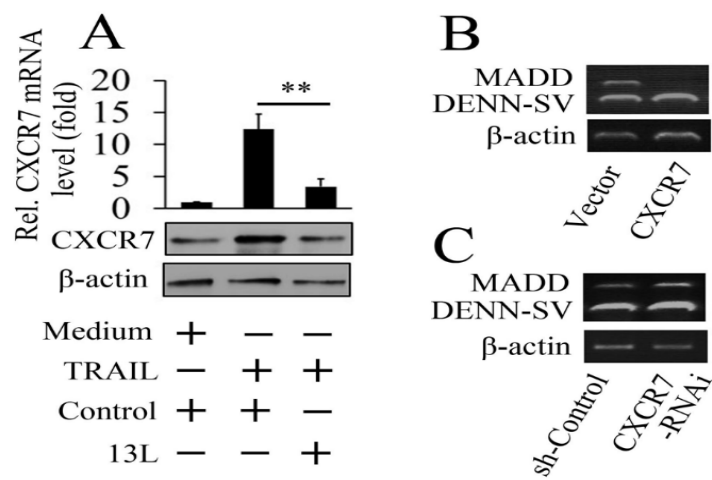

Figure 3. The Relationship between MADD and CXCR7. (A) T47D cells were transduced with the indicated virus for 48 hour, then treat with TRAIL for 24 hour at a concentration of $10 \mathrm{ng} / \mathrm{ml}$. CXCR7 mRNA (upper panel) and protein (lower panel) were detected by real-time RT-PCR and western blot, respectively. (B) T47D cells were transfected with empty vector or pcDNA3.1-CXCR7 for 48 hours. RNA was extracted and used for RT-PCR using F2-B2 primers. (C) Experiments were performed as in B except T47D cells were transfected with shRNA-control or CXCR7-RNAi\#1. All experiments were repeated at least three times with similar results. Bar graphs represent means $\pm \mathrm{SD}, \mathrm{n}=3(* * \mathrm{P}<0.01$; $* \mathrm{P}<0.05)$ 

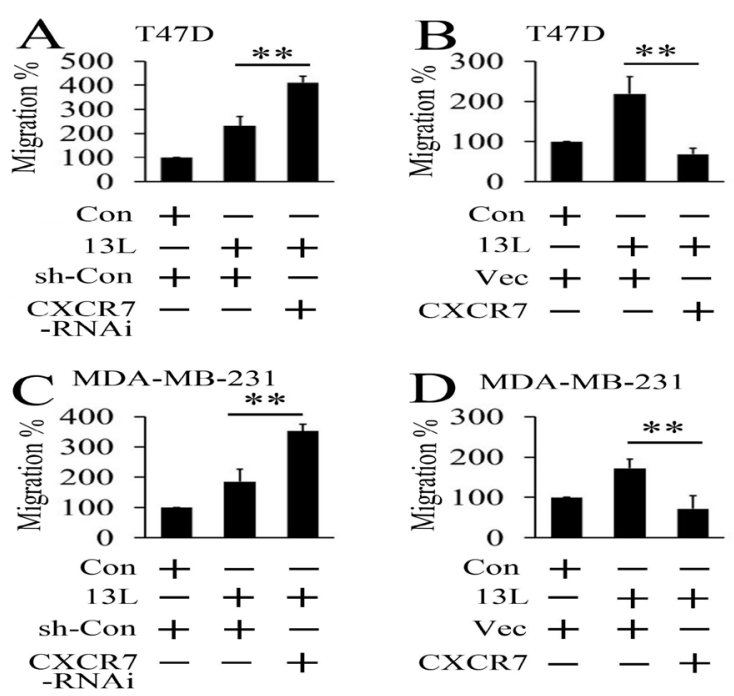

Figure 4. Determination of the Effect of CXCR7 on the Regulation of MADD-suppressed Breast Cancer Cells Migration. (A) T47D cells were transduced with the indicated virus (13L or control) for 24 hour, then transfected with siRNA-control or indicated siRNA-CXCR7s for 30 hours and allowed to migrate towards serum for 18 hours. (B) T47D cells were transduced with the indicated virus for 24 hour, then transfected with empty vector or pcDNA3.1-CXCR7 for 30 hours and allowed to migrate towards serum for 18 hours. (C, D) Experiments were performed as in A and B except MDAMB-231 cells were used. All experiments were repeated at least three times with similar results. Bar graphs represent means $\pm \mathrm{SD}$, $\mathrm{n}=3(* * p<0.01 ; * p<0.05)$

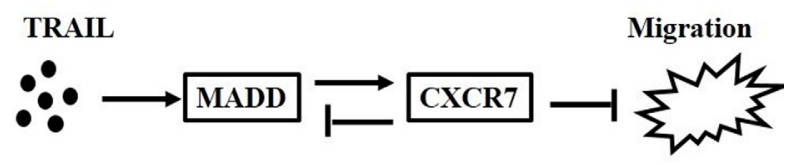

Figure 5. A Model for Regulation of TRAIF Suppressed Migration by MADD and CXCR7

transfection with CXCR7 RNAi plasmids synergistically promote T47D cells migration. Conversely, lower levels of migration were present in T47D cells when overexpression of CXCR7 (Figure 4B). To determine whether CXCR7mediated MADD-reduced migration is a common feature in breast cancer cells, similar experiments were performed in MDA-MB-231 cells (Figure 4C and D). All of these results demonstrate that CXCR7 play an important role in MADD reduced breast cancer cells migration.

\section{Discussion}

In this study, we provide a previously undescribed mechanism for TRAIL regulated breast cancer cell migration (Figure 5). We determined that TRAIL induces MADD and CXCR7 expression, result in decreasing breast cancer cell migration (Figure 5). Further, studies showed that MADD is an upstream regulatory factor of TRAILtriggered CXCR7 production; and conversely, CXCR7 attenuated MADD expression (Figure 5).

TRAIL, an Apo2 ligand, is a member of the cytokine tumor necrosis factor (TNF) superfamily, which induces apoptosis in a wide variety of tumors without harming normal cells (Riddick et al., 2013). So, TRAIL-based therapy is currently evaluated in clinical studies. However, other than activating canonical caspase-dependent apoptosis result in killing cancerous cells, TRAIL also can activate non-canonical (non-apoptotic) signal pathways, which is counterproductive for therapy (Srivastava et al., 2010). Thus, it is a critical question in clinical trials to explore the mechanism of TRAIL-mediated non-apoptotic signal pathways. In the present study, for the first time, we observed that TRAIL exert an anti-metastasis function through regulation of MADD and CXCR7 expression in breast cancer cells. Interesting, a previous study shown that TRAIL-induced miR-146a expression suppresses CXCR4-mediated human breast cancer migration (Wang et al., 2013). Our data provide another new insight into the non-apoptotic function of TRAIL in the prevention of human breast cancer migration.

In human, because of undergoing alternative splicing, many genes encode multiple isoforms result in the expression of related proteins, which have distinct biological function (Smith and Valcarcel, 2000). The IG20 is one of such genes and encode at least four different splice variants (SV), namely IG20, IG20-SV2, MADD/DENN, and DENN-SV, which is distinguished by differential splicing of exons 13L, 16, 21, 26 and 34 (Efimova et al., 2004). All IG20 gene are found up-regulated in multiple tumor types relative to their normal counterparts (Mulherkar et al., 2006). Due to its overexpression in cancer cells, more and more studies have tried to reveal the role of IG20 gene in the biological behavior of cancers. IG20 enhances their susceptibility to TNFa-induced apoptosis, whereas DENN-SV confers resistance to apoptosis (Efimova et al., 2004). MADD/DENN promote cancer cell survival and inhibit doxorubicin and TRAIL induced apoptosis in breast cancer cells (Mulherkar et al., 2006). Although the IG20 gene has been implicated in cancer cell survival and apoptosis in some studies, a role for IG20 gene in cancer migration has not been established. In this study, we showed that TRAIL treatment induced CXCR7 expression and MADD knock-down weaken TRAIL inhibited migration in breast cancer cells (Figure 1). Other isoforms of the IG20 gene may also be involved in cell migration. Further studies are needed to verify these putative functions.

The function of CXCR7 is interesting and controversial. CXCR7 was overexpressed in various tumors, including pancreatic cancer, lung cancer, prostate cancer and breast cancer, which suggest CXCR7 has important role in cancer progression (Zheng et al., 2010). It has been demonstrated that expression of CXCR7 influences experimental lung metastasis and promotes tumor growth (Iwakiri et al., 2009). In addition, Zabel BA et al. have shown that CXCR7 can regulate CXCL12-mediated migratory cues, lead to inhibiting CXCR4-mediated tumor cell transendothelial migration (Zabel et al., 2009). Another study has revealed that CXCR7 participated in regulation of motility and adhesion, MMP expression and directional chemotaxis in rhabdomyosarcoma cell (Grymula et al., 2010). On other hand, some studies suggested that CXCR7 can not activate intracellular signaling cascades, such as PI3K and ERK pathways, and is a non-signaling decoy receptor (Zheng et al., 2010). In this study, we found that TRAIL stimulation increased CXCR7 mRNA and protein 
Rui Wang and Jincheng $\mathrm{Li}$

levels in breast cancer cells. Further studies elucidated that CXCR7 is involved in TRAIL-regulated cell migration. These results are consistent with above studies showing that CXCR7 mediates tumor cell metastasis (Iwakiri et al., 2009; Zabel et al., 2009). Therefore, we suspected that the controversial biological effects elicited by CXCR7 may depend on cell type. Further studies should clarify roles of CXCR7 in migration and signaling cascades activated by CXCR7 in many other cancer cells.

In summary, our present study provides novel evidence showing that MADD and CXCR7 plays a functional role in regulating TRAIL-inhibited migration in the breast cancer cells. Although more studies are needed to understand the delicate regulatory mechanisms of breast cancer cell migration the obtained results may shed new lights on the understanding of the cancer resistance to therapy.

\section{References}

Aiuti A, Webb J, Bleul C, et al (1997). The chemokine SDF1 is a chemoattractant for human CD34+ hematopoietic progenitor cells and provides a new mechanism to explain the mobilization of CD34+ progenitors to peripheral blood. $J$ Exp Med, 185, 111-20.

Al-Zoubi AM, Efimova EV, Kaithamana S, et al (2001). Contrasting effects of IG20 and its splice isoforms, MADD and DENN-SV, on tumor necrosis factor alpha-induced apoptosis and activation of caspase-8 and -3.J Biol Chem, 276, 47202-11.

Attar R, Sajjad F, Qureshi MZ, et al (2014). TRAIL based therapy: overview of mesenchymal stem cell based delivery and miRNA controlled expression of TRAIL. Asian Pac J Cancer Prev, 15, 6495-7.

Azijli K, Weyhenmeyer B, Peters GJ, et al (2013). Noncanonical kinase signaling by the death ligand TRAIL in cancer cells: discord in the death receptor family. Cell Death Differentiation, 20, 858-68.

Azijli K, Yuvaraj S, Peppelenbosch MP, et al. (2012). Kinome profiling of non-canonical TRAIL signaling reveals RIP1Src-STAT3-dependent invasion in resistant non-small cell lung cancer cells. J Cell Science, 125, 4651-61.

Burns JM, Summers BC, Wang Y, et al (2006). A novel chemokine receptor for SDF-1 and I-TAC involved in cell survival, cell adhesion, and tumor development. The $J$ Experimental Med, 203, 2201-13.

Efimova EV, Al-Zoubi AM, Martinez O, et al (2004). IG20, in contrast to DENN-SV, (MADD splice variants) suppresses tumor cell survival, and enhances their susceptibility to apoptosis and cancer drugs. Oncogene, 23, 1076-87.

Falschlehner C, Emmerich CH, Gerlach B, et al (2007). TRAIL signalling: decisions between life and death. Int J Biochem Cell Biology, 39, 1462-75.

Grymula K, Tarnowski M, Wysoczynski M, et al (2010). Overlapping and distinct role of CXCR7-SDF-1/ITAC and CXCR4-SDF-1 axes in regulating metastatic behavior of human rhabdomyosarcomas. Int J Cancer, 127, 2554-68.

Hernandez L, Magalhaes MA, Coniglio SJ, et al (2011). Opposing roles of CXCR4 and CXCR7 in breast cancer metastasis. Breast Cancer Res, 13, 128.

Iwakiri S, Mino N, Takahashi T, et al (2009). Higher expression of chemokine receptor CXCR7 is linked to early and metastatic recurrence in pathological stage I nonsmall cell lung cancer. Cancer, 115, 2580-93.

Kakinuma T, Hwang ST (2006). Chemokines, chemokine receptors, and cancer metastasis. J Leukocyte Biol, 79, 639-51.

Kruyt FA (2008). TRAIL and cancer therapy. Cancer Letters, 263, 14-25.

Kurada BR, Li LC, Mulherkar N, et al (2009). MADD, a splice variant of IG20, is indispensable for MAPK activation and protection against apoptosis upon tumor necrosis factoralpha treatment. J Biological Chemistry, 284, 13533-41.

Mulherkar N, Ramaswamy M, Mordi DC, et al (2006). MADD/ DENN splice variant of the IG20 gene is necessary and sufficient for cancer cell survival. Oncogene, 25, 6252-61.

Nogueira DR, Yaylim I, Aamir Q, et al (2014). TRAIL mediated signaling in pancreatic cancer. Asian Pac J Cancer Prev, 15, 5977-82.

Pablos JL, Amara A, Bouloc A, et al (1999). Stromal-cell derived factor is expressed by dendritic cells and endothelium in human skin. Am J Pathol, 155, 1577-86.

Riddick E, Evans S, Rousch J, et al (2013). Identification of death receptors DR4 and DR5 in HTB-12 astrocytoma cell lines and determination of TRAIL sensitivity. J Solid Tumors, 3, 20-26.

Schievella AR, Chen JH, Graham JR, et al (1997). MADD, a novel death domain protein that interacts with the type 1 tumor necrosis factor receptor and activates mitogenactivated protein kinase. J Biol Chem, 272, 12069-75.

Shi J, Zheng D, Man K, et al (2003). TRAIL: a potential agent for cancer therapy. Current molecular medicine, 3, 727-736.

Smith CW, Valcarcel J (2000). Alternative pre-mRNA splicing: the logic of combinatorial control. Trends Biochem Sci, 25, 381-8.

Srivastava RK, Kurzrock R, Shankar S (2010). MS-275 sensitizes TRAIL-resistant breast cancer cells, inhibits angiogenesis and metastasis, and reverses epithelial-mesenchymal transition in vivo. Molecular Cancer Therapeutics, 9 , 3254-66.

Szliszka E, Mazur B , Zydowicz G, et al (2009). TRAIL-induced apoptosis and expression of death receptor TRAIL-R1 and TRAIL-R2 in bladder cancer cells. Folia Histochem Cytobiol, 47, 579-85.

Tachibana K, Hirota S, Iizasa H, et al (1998). The chemokine receptor CXCR4 is essential for vascularization of the gastrointestinal tract. Nature, 393, 591-4.

Thelen M, Thelen S (2008). CXCR7, CXCR4 and CXCL12: an eccentric trio? J Neuroimmunol, 198, 9-13.

Turner A, Li LC, Pilli T, et al (2013). MADD knock-down enhances doxorubicin and TRAIL induced apoptosis in breast cancer cells. PloS One, 8, 56817.

Wang D, Liu D, Gao J, et al (2013). TRAIL-induced miR-146a expression suppresses CXCR4-mediated human breast cancer migration. The FEBS J, 280, 3340-53.

Wang H, Xu C, Kong X, et al (2014). Trail resistance induces epithelial-mesenchymal transition and enhances invasiveness by suppressing PTEN via miR-221 in breast cancer. PloS One, 9, 99067.

Zabel BA, Wang Y, Lewen S, et al (2009). Elucidation of CXCR7-mediated signaling events and inhibition of CXCR4-mediated tumor cell transendothelial migration by CXCR7 ligands. J Immunol, 183, 3204-11.

Zheng K, Li HY, Su XL, et al (2010). Chemokine receptor CXCR7 regulates the invasion, angiogenesis and tumor growth of human hepatocellular carcinoma cells. J Exp Clin Cancer Res, 29, 31 . 